\title{
Isolated Hepatic Tuberculosis - Serohepatic Type
}

\author{
Dr. Prem Gowtham.E ${ }^{1}$,Dr. Kalaichezhian. $\mathrm{M}^{2}$,Dr. Prabakaran.M ${ }^{3}$. \\ ${ }^{1}$ Post Graduate Resident, Department of Radiodiagnosis, Sree Balaji Medical College and Hospital, \\ Chromepet, Chennai. \\ ${ }^{2}$ Assistant Professor, Department of Radiodiagnosis, Sree Balaji Medical College and Hospital, \\ Chromepet, Chennai. \\ ${ }^{3}$ Professor and HOD, Department of Radiodiagnosis, Sree Balaji Medical College and Hospital, \\ Chromepet, Chennai.
}

\begin{abstract}
Though tuberculous infection constitutes one of the more common causes of death and morbidity in the developing world, hepatic tuberculosis is a rare presentation. Hepatic involvement, usually in the form of multiple small-sized miliary nodules, is seen along with other organ involvement. In contrast, isolated hepatic tuberculosis is seldom encountered in clinical practice. In this article we highlight about the serohepatic tuberculosis which is a rare presentation among other hepatic tuberculosis types. Uniquely, the patient had sub capsular as well as extra-capsular involvement. Diagnosis involved imaging and histopathological examination. Keyword: USG, CT, Tuberculoma, Hepatic tuberculosis.
\end{abstract}

\section{Introduction}

Hepatic tuberculosis commonly affects young age group. It is commonly seen in second decade of life with male: female ratio of 2: 1 . Hepatic tuberculosis can be of primary or secondary. Levine classified into five types namely miliary, concomitant hepatic and pulmonary disease, primary hepatic tuberculosis, hepatic abscess and tubercular cholangitis [1]. Whereas according to pathology $\mathrm{Yu}$ et al classified into three types namely parenchymal which is additionally sub divided into micro and macro nodular, serohepatic and tubercular cholangitis [2]. In this journal we discuss about the serohepatic tuberculosis with sub capsular, extra capsular and macronodular tuberculosis.

\section{Case Report}

A 19-year boy presented to the Gastroenterology OPD with the complaints of upper abdominal pain for past 3 days. There was history of intermittent fever, poor appetite and weight loss for the past 3 months. There was no history of vomiting or jaundice. There was no other significant past medical history. On examination patient is lean and afebrile. Mild tenderness was noted over epigastric region. Complete blood count was within normal limits. Liver function test showed increased serum alkaline phosphatase. Serum bilirubin level was within normal limits.

Ultrasound abdomen showed mild hepatomegaly and thrombosis of left portal vein [Figure 1]. There was reduction in volume of left lobe of liver consisting with heterogeneous echotexture and multiple parenchymal calcifications. Ultrasound showed splenomegaly and enlarged node in the porta.
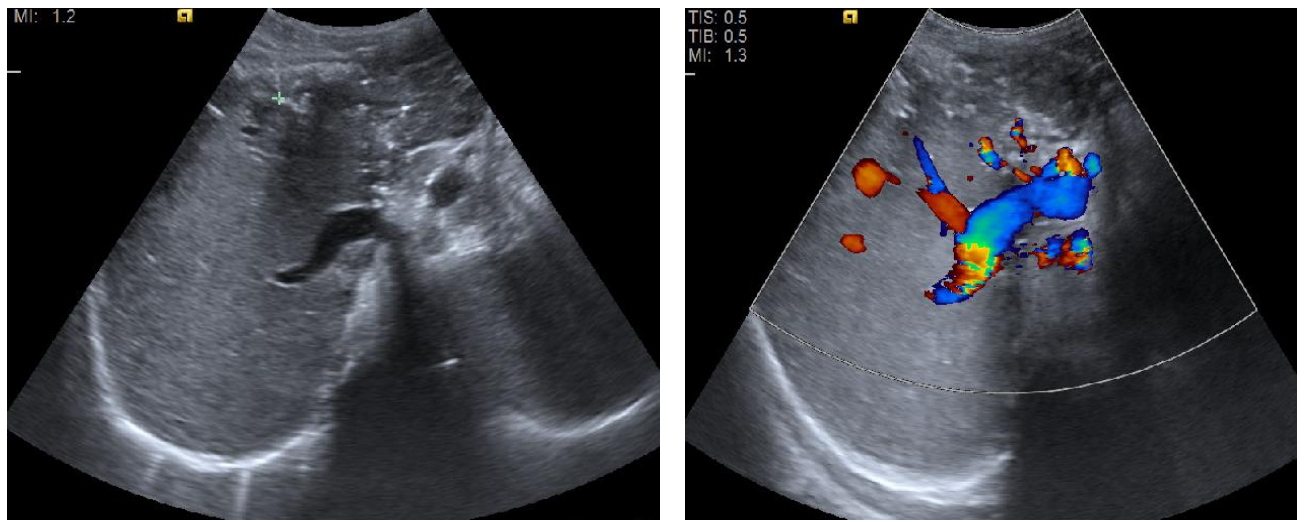

Contrast enhanced computerized tomography (CECT) of abdomen showed hepatomegaly with large heterogeneously enhancing area measuring $10.3 \times 10.5 \mathrm{~cm}$ with lobulated margins involving both lobes of liver. It contained multiple small peripherally enhancing hypodense nodules and multiple calcifications seen almost 
replacing the left lobe of liver [Figure 2]. Similar smaller lesions were noted in the right lobe of liver and the caudate lobe. Some of the lesions in the right lobe are in sub capsular location and extra capsular region.
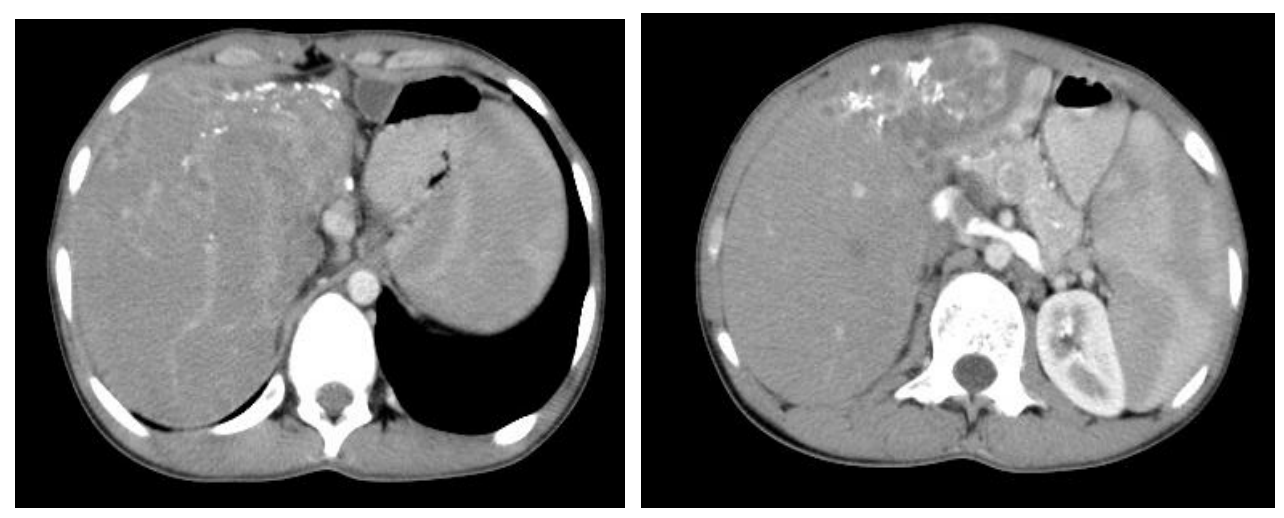

Other findings include dilatation of main portal vein $(14 \mathrm{~mm})$ and previous thrombosis of left portal vein due to possible involvement by the left lobe lesion. Prominent hepatic artery branches were seen in the course of obliterated left portal vein. Multiple peri portal and peri pancreatic nodes were seen. There was splenomegaly due to portal hypertension secondary to left portal vein thrombosis. Upper GI endoscopy shows single prominent vein in oesophagus. Ultrasound guided trucut biopsy showed liver tissue with loss of architecture. The portal areas showed dense lymphocytic infiltrate. The lobules showed multiple caseating epithelioid cells, Langerhans's giant cells and sparse lymphocytes surrounding it. Rest of liver tissue shows extensive destruction of architecture due to extensive fibrosis, pseudo lobule formation, diffuse lymphocyte infiltrate in the sinusoids and in around the pseudo lobule. HPE features were suggestive of tuberculosis.

\section{Discussion}

Liver is a common site for granuloma formation owing to its rich blood supply, lying at the distal end of portal circulation and large number of reticuloendothelial cells. Usually the patients are asymptomatic. Hepatic tuberculosis is rare because of low oxygen tension in the liver which is unfavourable for growth of mycobacterium [3]. Hepatic tuberculosis secondary to pulmonary or intestinal tuberculosis is more common. The diagnosis of hepatic tuberculosis depends upon the imaging and histopathology report. Imaging techniques USG and CT are useful in making the diagnosis of hepatic tuberculosis. The typical CT findings of hepatic tuberculosis include multiple lesions of varying density, indicating that there are lesions in different pathologic stages coexisting in hepatic TB, including tuberculous granuloma, liquefaction necrosis, fibrosis or calcification [2]. Hepatic tuberculosis can be of primary or secondary. Levine classified into five types namely miliary, concomitant hepatic and pulmonary disease, primary hepatic tuberculosis, hepatic abscess and tubercular cholangitis [1]. Whereas according to pathology $\mathrm{Yu}$ et al classified into three types namely parenchymal which is additionally sub divided into micro and macro nodular, serohepatic and tubercular cholangitis [2]. USG and CT study may show intrahepatic hypodense lesions with or without associated high-density calcific lesions. CECT may reveal variable enhancement. Cao et al, reported that contrast-enhanced ultrasonography be helpful in differentiating the diagnosis of hepatic TB from other hepatic focal lesions [4]. In recent years, the F-18 FDG PET/CT is widely used in the diagnosis and staging of malignant tumours, but prone to misdiagnosis in the diagnosis of hepatic tuberculosis, because the hepatic tuberculosis also showed FDG-avid [5]. Differential diagnoses for hepatic tuberculosis on PET CT include necrotic tumours such as cholangiocarcinoma, hepatocellular carcinoma and metastatic carcinoma. Serohepatic tuberculosis is a rare presentation and mostly involves the subserosal plane of the liver. i.e connective tissue lying beneath the serous coat of the liver which is inseparable from the fibrous glisson capsule. The imaging features include peripheral placed lesions in the sub capsular plane of the liver. The thickened liver capsule and sub-capsule overlying the lesions simulates a 'sugar coating' commonly referred to 'frosted liver'. In this patient, the lesions were seen in sub capsular as well as extra capsular location

\section{Conclusion}

In conclusion, hepatic tuberculosis is usually associated with symptom less and usually diagnosed as incidental finding. Diagnosis involves both the imaging and aspiration cytology. Some of the key points to suspect hepatic tuberculosis may include (a) past history of tuberculosis or contact with tuberculosis, fever, fatigue, weight loss, abdominal pain, jaundice, liver tenderness (b) Elevated Serum levels such as alkaline phosphatase (c) enhancing nodules and calcifications in intrahepatic / sub capsular and peri capsular location on CECT scan. 


\section{Reference}

[1]. Levine C (1990) Primary macronodular hepatic tuberculosis: US and CT appearances Gastrointest Radiol 15: 307 - 309.

[2]. Yu RS, Zhang SZ, WU JJ et al (2004) imaging diagnosis of 12 patient with hepatic tuberculosis. World J Gastroenterol 10(11): $1639-1642$.

[3]. Singh S, Jain P, Aggarwal G, Dhiman P, Singh S and Sen R. Primary hepatic tuberculosis: A rare but fatal clinical entity if undiagnosed. Asian Pac J Trop Med 2012; 5: 498-9

[4]. Cao BS, Li XL, Li N and Wang ZY. The Nodular Form of Hepatic Tuberculosis Contrast-Enhanced Ultrasonographic Findings With Pathologic Correlation. J Ultrasound Med 2010; 29: 881-8.

[5]. Wang YT, Lu F, Zhu F, Qian ZB, Xu YP and Meng T. Primary hepatic tuberculoma appears similar to hepatic malignancy on F-18 FDG PET/CT. Clin Nucl Med 2009; 34: 528-9 